\title{
Depresión y disminución de la función cognitiva en adultos mayores de un hospital peruano, 2010-2015
}

\section{Depression and cognitive function decay in elderly subjects in a Peruvian hospital, 2010 - 2015}

Correspondencia Marwy Valer Quispe marhanji@gmail.com

Recibido: 23/06/2018 Aprobado: 18/07/2018

Citar como: Apaza J, Valer $M$, Runzer-Colmenares FM. Depresión y disminución de la función cognitiva en adultos mayores de un hospital peruano, 2010-2015. Acta Med Peru. 2018;35(3):191-2
Jessica Apaza ${ }^{1, a}$, Marwy Valer ${ }^{1, a}$, Fernando M. Runzer-Colmenares ${ }^{1, b}$

1 Universidad Científica del Sur. Lima, Perú.

a Estudiante de Medicina Humana, b Médico geriatra

\section{Sr. Editor:}

Los problemas afectivos suelen subestimarse y son abordados inadecuadamente en los adultos mayores (AM). Se estima para el año 2020 la depresión será la segunda causa de años de vida saludable perdidos; en el Perú, los esfuerzos para afrontarla, que incluyen algunos con un enfoque multisectorial, son aún aislados $^{[1]}$.

Los trastornos neurocognitivos (TNC) difieren del envejecimiento cognitivo fisiológico; cursan con amnesia retrógrada/anterógrada y afasia anómica, afectan la atención y memoria inmediata y provocan síntomas afectivos con alteración de la funcionalidad, principal característica que los diferencia de los problemas cognitivos fisiológicos. Según estudios epidemiológicos, más del $20 \%$ de AM cursa con algún problema de salud mental como depresión o TNC, con diversos grados de severidad ${ }^{[2]}$.

La depresión asociada a TNC es un problema neuropsiquiátrico deletéreo frecuente que genera deterioro funcional, social y familiar, además de altos índices de morbimortalidad. Además, se ha demostrado que el padecer de depresión es un factor de riesgo para desarrollar TNC ${ }^{[1]}$.

La asociación entre depresión y TNC suscita cada vez un mayor interés, aun cuando resulta un reto determinar si el paciente padece un problema afectivo que provoca el TNC o coexisten. Por ello, existen diversas escalas cognitivas, como las de valoración geriátrica integral, que son útiles para identificar las funciones mentales afectadas y determinar si los TNC diagnosticados podrían revertirse luego de tratar la depresión, lo cual es útil en la toma de decisiones diagnósticas y terapéuticas.

En el presente estudio mostramos el análisis preliminar de cuatro diferentes escalas cognitivas aplicadas en AM con el objetivo de evaluar la disminución de funciones cognitivas según presencia de estados depresivos previos. Como parte de una cohorte evaluada en el Centro Médico Naval del Perú entre 2010 y 2015 se analizaron datos de 657 AM sin antecedente de TNC ${ }^{[3]}$. Se evaluó, al inicio y final del seguimiento, a los participantes con las siguientes escalas: Montreal Cognitive Assessment (MoCA), versión en español, MiniMental State Examination, versión peruana, Cuestionario de Pfeiffer, versión en español y test del reloj, versión Shulman.

Se analizaron las diferentes medidas en participantes con y sin antecedente de depresión, determinada al inicio del seguimiento ${ }^{[4]}$. Como se aprecia en la Tabla 1, para las cuatro escalas, se obtuvieron promedios menores en los participantes con antecedente de depresión, al inicio del seguimiento. Además, se observó que la disminución de funciones cognitivas al final del seguimiento fue mayor en los participantes con antecedente de depresión, diferencia que fue más grande usando la escala MoCA que evalúa la capacidad 
Tabla 1. Análisis comparativo de disminución de función cognitiva según antecedentes de depresión ( $n=657)$.

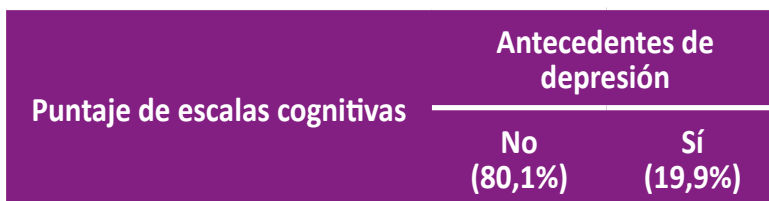

Al inicio del seguimiento $(x \pm D E)$

$\begin{array}{lcc}\text { MMSE } & 28,02 \pm 0,21 & 26,12 \pm 1,16 \\ \text { MoCA } & 25,04 \pm 0,67 & 23,57 \pm 1,48 \\ \text { Cuestionario de Pfeiffer } & 8,08 \pm 0,08 & 6,97 \pm 1,09 \\ \text { Test del reloj de Shulman } & 9,61 \pm 0,08 & 8,07 \pm 1,03 \\ \text { Al final del seguimiento }(x \pm D E) & & \\ \text { MMSE } & 23,09 \pm 0,84 & 16,47 \pm 3,84 \\ \text { MoCA } & 21,68 \pm 2,73 & 13,49 \pm 2,82 \\ \text { Cuestionario de Pfeiffer } & 6,55 \pm 0,18 & 4,48 \pm 1,73 \\ \text { Test del reloj de Shulman } & 5,32 \pm 0,75 & 4,56 \pm 1,69\end{array}$

x: media; DE: desviación estándar; MMSE: MiniMental state examination; MoCA: Montreal cognitive assessment

visuoespacial, capacidad ejecutiva, memoria, atención, lenguaje, capacidad de abstracción y orientación, dominios que se pueden afectar en TNC secundarios a procesos afectivos en AM.

Se concluye que para evaluar correctamente a pacientes con TNC se debe considerar la evaluación afectiva, pues se observó que el antecedente de depresión acelera el deterioro cognitivo $y$, escalas de tamizaje como MoCA, podrían ser opciones acertadas donde se podría tener en cuenta a la depresión como factor de riesgo potencialmente modificable de TNC ${ }^{[5]}$.

Fuente de financiamiento: No se recibió ningún apoyo económico.

Conflictos de intereses: Todos los autores declaran que no tienen ningún conflicto de interés en relación con este artículo.

\section{REFERENCIAS BIBLIOGRÁFICAS}

1. Jonis M, Llacta D. Depresión en el adulto mayor, cual es la verdadera situación en nuestro país. Rev Med Hered. 2013;24(1):78-9.

2. American Association for Geriatric Psychiatry. Geriatrics and mental health - the facts [Internet]. McLean, VA: AAGP; 2012 [citado el 26 de mayo de 2013]. Disponible en: www.aagponline.org/prof/facts mh.asp

3. Sandival GA, Runzer-Colmenares FM, Parodi JF. La valoración funcional como predictor de deterioro cognitivo: Cohorte retrospectiva. Medwave. 2017;17(9):e7099. doi: 10.5867/ medwave.2017.09.7099.

4. Parodi JF, Nieto-Gutierrez W, Tellez WA, Ventocilla-Gonzales I, Runzer-Colmenares FM, Taype-Rondan A. Velocidad de marcha y desarrollo de trastornos neurocognitivos en adultos mayores: resultados de una cohorte peruana. Rev Esp Geriatr Gerontol. 2017;53(2):63-118.

5. Almeida OP, Hankey GJ, Yeap BB, Golledge J, Flicker L. Depression as a modifiable factor to decrease the risk of dementia. Transl Psychiatry. 2017;7(5):e1117.

\title{
Ahora puede enviar sus artículos para Acta Médica Peruana en nuestro Open Journal System:
}

\author{
www.amp.cmp.org.pe
}

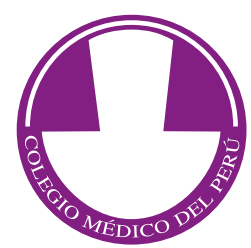

